\title{
Two-photon free-induction decay with electromagnetically induced transparency
}

\author{
J. F. Chen, Weixin Lu, Shuyuan Wang, M. M. T. Loy, G. K. L. Wong, and Shengwang Du* \\ Department of Physics, The Hong Kong University of Science and Technology, \\ Clear Water Bay, Kowloon, Hong Kong, China \\ ${ }^{*}$ Corresponding author: dusw@ust.hk
}

(Submitted to Optics Letters on December 21, 2009; revised March11, 2010; accepted May 11, 2010; published May $28,2010)$

Compiled August 9, 2010

We report the observation of coherent two-photon free-induction decay (FID) in a three-level $\Lambda$ system excited by a low-intensity square-modulated laser pulse. Using electromagnetically induced transparency in a ${ }^{85} \mathrm{Rb}$ cold atomic cloud, for the first time, we observe FID signals with coherence time exceeding the relevant atomic excited-state natural lifetime. Due to the on-resonance enhancement, the two-photon FID signal can be observed at the falling edge without using heterodyne means. (C) 2010 Optical Society of America

OCIS codes: 190.4180, 020.1670.

Transient optical phenomena associated with coherent interaction of radiation field with matter have been a fascinating aspect of the field of atomic, molecular and optical physics. These fundamental studies have led to the development of many powerful analytical tools, such as nuclear magnetic resonance (NMR) spectroscopy, atomic clock, etc. Following the invention of the laser, there was much interest in optical coherent transitions from a twolevel system, which is formally equivalent to the well studied NMR [1]. This led to the realization of optical analogs of spin resonance effects such as nutation and free-induction decay (FID) [2,3]. Soon afterwards, twophoton nutation and FID in three-level systems were studied and demonstrated [4-8]. Recently, FIDs have also been reported in cold atoms $[9,10]$, and linked to the phenomenon of optical precursors [11].

However, all two-photon FID experiments in the past focused on three-level ladder schemes. As a result, the FID temporal duration is limited by the atomic lifetimes of the excited states. Meanwhile, to avoid single-photon absorption, the applied laser fields are often far detuned from the intermediate states. Therefore, the two-photon FID signals are always very weak and can only be indirectly detected by heterodyne means through interference between radiation and the driving field.

In this Letter, we report the observation of coherent two-photon FIDs in a three-level $\Lambda$ system excited by a weak square-modulated laser pulse. Using electromagnetically induced transparency (EIT) [12] in a ${ }^{85} \mathrm{Rb}$ cold atomic cloud, the two-photon coherence between the two ground states is very long. Moreover, the elimination of single-photon on-resonance linear absorption allows us to tune both two driving laser fields on atomic resonances to enhance the two-photon interaction. As a result, we are able to demonstrate for the first time direct observation of two-photon FIDs at the falling edge of the square pulse without having to use heterodyne means. We ob-
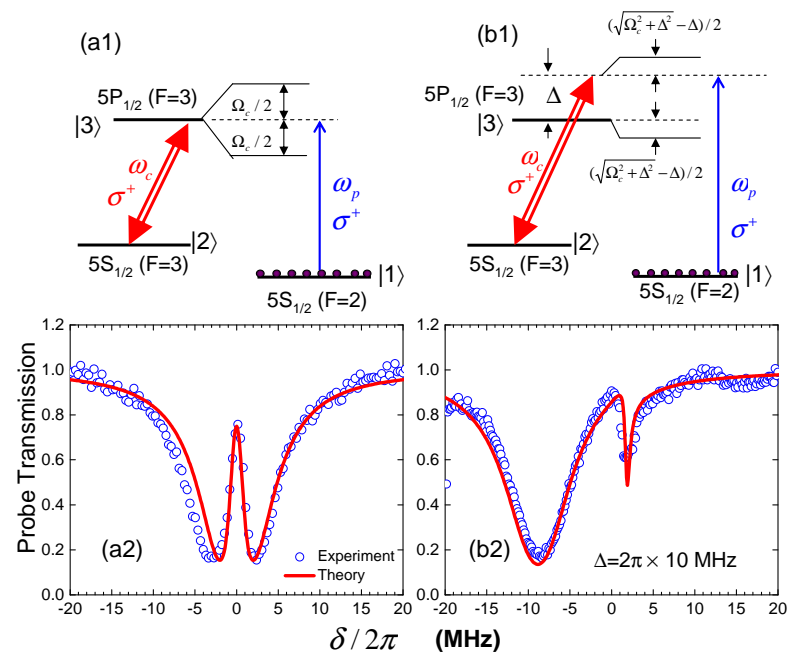

Fig. 1. (color online). Schematics of ${ }^{85} \mathrm{Rb}$ energy levels for (a1) on-resonance and (b1)off-resonance EIT systems. (a2) and (b2) are the measured on-resonance and off-resonance EIT transmission as functions of the twophoton detuning $\delta$, taken at an optical depth $\alpha_{0} L=2$ and coupling Rabi frequency $\Omega_{c}=1.4 \gamma_{13}$.

tain FID signals with a temporal length more than four times longer than the atomic lifetime of the excited state.

We work with ${ }^{85} \mathrm{Rb}$ cold atoms in a two-dimensional (2D) magneto-optical trap (MOT) at a low optical depth of about 2. Schematics of the three-level $\Lambda$ EIT system is shown in Fig. 1(a1) and (b1). A coupling laser $\left(\omega_{c}\right)$ is applied to the transition $|2\rangle \rightarrow|3\rangle$, and a weak probe laser $\left(\omega_{p}\right)$ to the transition $|1\rangle \rightarrow|3\rangle . \Delta$ denotes the coupling laser detuning. The two-photon detuning is defined as $\delta=\omega_{p}-\omega_{c}-\Delta \omega_{21}$, where $\Delta \omega_{21}$ is the energy difference between $|1\rangle$ and $|2\rangle$. When the coupling laser is on resonance $[\Delta=0$, Fig. 1(a1)], the EIT transparency occurs at the probe single-photon resonance, as shown in the 
probe transmission spectrum in Fig. 1(a2). In the offresonance EIT case with $\Delta=2 \pi \times 10 \mathrm{MHz}$ in Fig. 1(b1) and (b2), the induced transparency lies also at $\delta=0$. In both cases when the two-photon detuning is zero, the two-photon coherence time can be very long because of the two long-lived ground states.

The experimental apparatus has been described in our recent work [13]. The 2D MOT has a length $L=1.5 \mathrm{~cm}$ and a temperature of about $100 \mu \mathrm{K}$. We periodically run the system with a MOT trapping time of $4.5 \mathrm{~ms}$ followed by a measurement window of $0.5 \mathrm{~ms}$. At the end of the trapping time, all the atoms are optically pumped to the ground level $|1\rangle$. During the measurement window when the probe and coupling lasers are on, all the MOT laser beams are switched off. The MOT magnetic field has a transverse gradient of 10 Gauss/cm and remains on all the time. The coupling laser is aligned collinearly with a small angle of $2^{o}$ respect to the probe beam. The weak square-modulated probe pulse propagates through the cold atoms and is detected by a photon-multiplier tube (PMT, Hamamatsu, H6780-20, 0.78 ns rise time). The data is recorded by a $1 \mathrm{GHz}$ realtime digital oscilloscope (Tektronix, TDS684B) and averaged over 30 traces.

We focus on two-photon FIDs excited by a weak step-modulated probe pulse. The probe optical field is described classically as $\frac{1}{2} E_{p}(z, t) e^{i\left[k_{p} z-\omega_{p} t\right]}+$ c.c. with $k_{p}=\omega_{p} / c$. With the probe laser that is sufficiently weak, the atomic population remains mostly at the ground state $|1\rangle$. This ground state approximation allows us to avoid the complexity of density matrix approach. Instead, we will study the atomic response using $|\Psi(t)\rangle=a_{1}(t)|1\rangle+a_{2}(t)|2\rangle+a_{3}(t)|3\rangle$. In the counter rotating reference frame, with $a_{1} \simeq 1$ and two-photon resonance condition $\delta=0$, we have the Schrodinger equations for the probability amplitudes $a_{2}$ and $a_{3}$ :

$$
\begin{aligned}
& \dot{a_{2}}=-\gamma_{12} a_{2}+i \frac{\Omega_{c}^{*}}{2} a_{3}, \\
& \dot{a_{3}}=i \frac{\Omega_{p}}{2}+i \frac{\Omega_{c}}{2} a_{2}+i\left(\Delta+i \gamma_{13}\right) a_{3},
\end{aligned}
$$

where $\Omega_{p}=E_{p} \mu_{31} / \hbar$ and $\Omega_{c}=E_{c} \mu_{32} / \hbar$ are the probe and coupling Rabi frequencies, respectively. $\mu_{i j}$ is the electric dipole matrix element between $|i\rangle$ and $|j\rangle \cdot \gamma_{13}=$ $2 \pi \times 3 \mathrm{MHz}$ is the electric dipole relaxation rate between $|1\rangle$ and $|3\rangle$. The two-photon dephasing rate between the two ground states $|1\rangle$ and $|2\rangle$ is measured to be $\gamma_{12}=$ $0.07 \gamma_{13}$. The probe wave propagation is governed by the slowly varying envelop equation :

$$
\frac{\partial E_{p}(z, t)}{\partial z}+\frac{1}{c} \frac{\partial E_{p}(z, t)}{\partial t}=\frac{i}{2} \frac{\omega_{p}}{c \varepsilon_{0}} P(z, t),
$$

where $P(z, t)=2 N \mu_{13} a_{3}$ is the induced electric dipole at the probe transition with atomic density $N$.

We first consider the transient response of the onresonance $(\Delta=0)$ EIT system from an input stepoff on-resonance pulse $E_{i n}(t)=E_{0} \Theta(-t)$, where $\Theta$ is the Heaviside function. At time $t=0$ before the probe field is switched off, the atoms are prepared in a dark

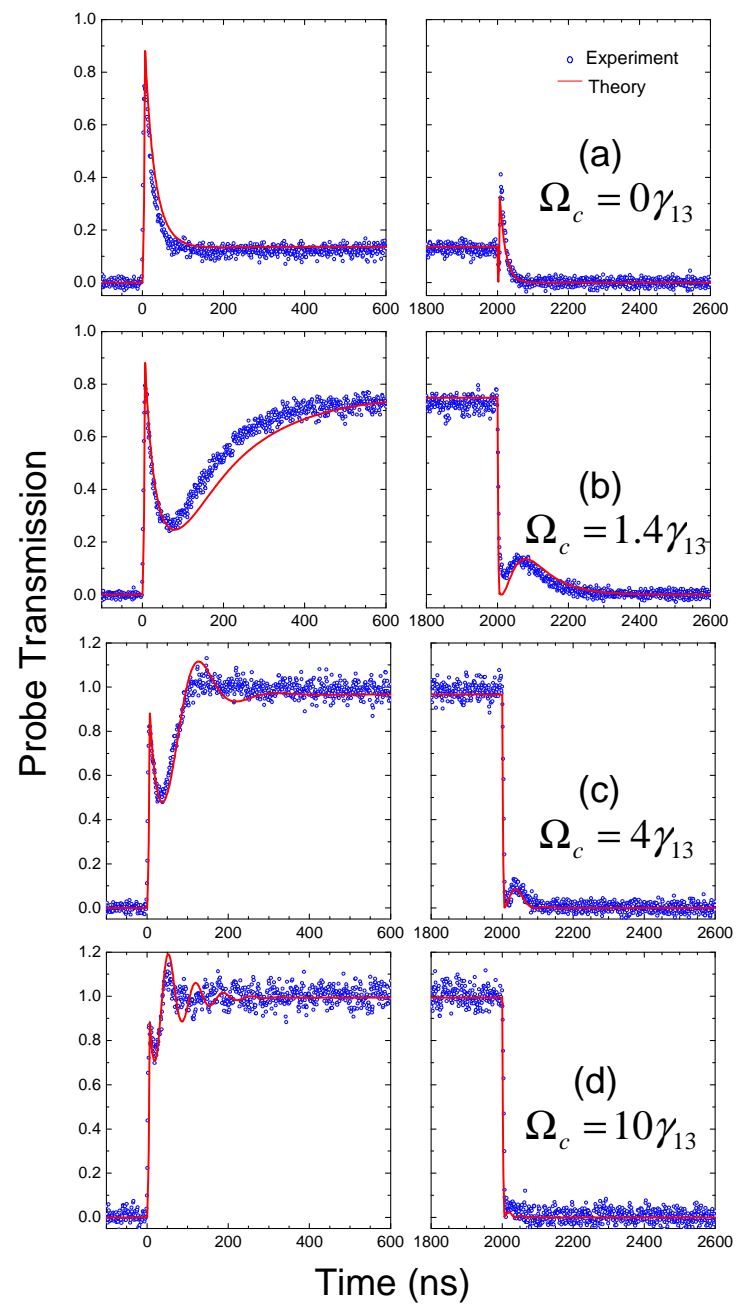

Fig. 2. (color online). Two-photon FIDs with onresonance $(\Delta=0)$ EIT excited by a weak square probe pulse driven by different coupling laser powers at an optical depth $\alpha_{0} L=2$.

state: $|\Psi(0)\rangle \simeq|1\rangle-\frac{\Omega_{p}}{\Omega_{c}}|2\rangle[14]$. With this initial condition, one can solve the master equation (1) by setting $\Omega_{p}(t>0)=0$ and obtain

$$
a_{3}(t>0)=\frac{-i \Omega_{p}}{\Omega_{e}} \sin \left(\frac{\Omega_{e} t}{2}\right) e^{-\left(\gamma_{13}+\gamma_{12}\right) t / 2},
$$

where the effective coupling Rabi frequency is defined as $\Omega_{e}=\sqrt{\left|\Omega_{c}\right|^{2}-\left(\gamma_{13}-\gamma_{12}\right)^{2}}$. At low optical depth when the propagation effect is negligible, Eq. (2) gives an approximate solution for the FID radiation field:

$$
\begin{aligned}
E_{f i d}(t) & \simeq \frac{i \omega_{p} L}{2 c \varepsilon_{0}} P(t) \\
& =\frac{\alpha_{0} L \gamma_{13}}{\Omega_{e}} E_{0} \Theta(t) \sin \left(\frac{\Omega_{e} t}{2}\right) e^{-\left(\gamma_{13}+\gamma_{12}\right) t / 2}(4)
\end{aligned}
$$

where $\alpha_{0}=N k_{p}\left|\mu_{13}\right|^{2} /\left(\varepsilon_{0} \hbar \gamma_{13}\right)$ is the on-resonance absorption coefficient. Equation (4) shows some interesting features about the two-photon FID. Similar to that in a two-level system, the FID field is proportional to the 
optical depth $\alpha_{0} L$ due to the collective coherent effect. However, different from the FID in the two-level system with an initial excited macroscopic electric dipole, the initial electric dipole of the EIT system is zero because the atoms are prepared in the dark state. After the probe laser is switched off, the stored two-photon coherence is released by the coupling laser. There are three characteristic times that determine the transient pattern. The first is the Rabi time $\tau_{r}=2 \pi / \Omega_{e}$ of the oscillation period. The second is the excited state dipole relaxation time $\tau_{13}=1 / \gamma_{13}=53 \mathrm{~ns}$. The third is the ground state dephasing time $\tau_{12}=1 / \gamma_{12}=758 \mathrm{~ns}$. Since $\tau_{12}$ is very long compared to other two times, the FID duration is determined by $\operatorname{Max}\left\{\tau_{r}, \tau_{13}\right\}$. The physics can be understood in the following two-photon process picture. As the coupling laser is weak, it takes very a long time to release the atoms from the dark state and the time scale is mainly determined by the coupling Rabi frequency. On the other hand, as the coupling laser becomes strong, the atoms are cycled between $|2\rangle$ and $|3\rangle$, and the leakage of photons to the transition $|3\rangle \rightarrow|1\rangle$ is determined by $\tau_{13}$. Therefore, the two-photon FID signal duration can be controlled by the coupling laser. The ultimate coherence time is limited by $\tau_{12}$.

The on-resonance $(\Delta=0)$ EIT experimental data (blue dots) of probe square pulse transmission with different coupling powers are shown in Fig. (2). The weak input square pulse has a length of $2 \mu \mathrm{s}$ and a rise (fall) time of $7 \mathrm{~ns}$. The solid red curves are obtained by numerically solving the atom-field coupled equations (1) and (2). When the coupling laser is off, Fig. 2(a) shows the FID in the two level system where the exponential decay is determined by $\gamma_{13}$. When we apply the coupling laser, the FID signals are no longer a single exponential decay curve. At $\Omega_{c}=1.4 \gamma_{13}$, the long transient duration of about 200 ns agrees with the Rabi time $\tau_{r}$ and is 4 times longer than $\tau_{13}$. As we further increase the coupling power, the transient signal becomes weaker and shorter. These behaviors can be explained in the dressedstate picture as shown in Fig. 1(a1) where the excited state is split into two with an energy separation of $\hbar \Omega_{c}$. The radiations from these two dressed states interfere and result in beating. We believe that the transient signals after the falling edge are the first direct observation of two-photon FID without using heterodyne means.

We then study two-photon FID in the off-resonance EIT system. Figure 3 shows the results with $\Delta=2 \pi \times 10$ $\mathrm{MHz}$ and $\Omega_{c}=1.4 \gamma_{13}$. Compared to that of the onresonance case in Fig. 2(b), the transient signal is much weaker and is barely detectable at the falling edge. The oscillation time of about $100 \mathrm{~ns}$ at the rising edge is well described by the effective Rabi time $2 \pi / \sqrt{\left|\Omega_{c}\right|^{2}+\Delta^{2}} \simeq$ $2 \pi / \Delta$. This can be explained again in the dressed-state picture, depicted in Fig. 1(b1).

While the observed FID signals are consistent with the theory, we note there is slight mismatching at a long time scale in Fig. 2(c),(d) and Fig. 3. As shown in the theoretical curves in Fig. 2(c),(d), the damped oscilla-

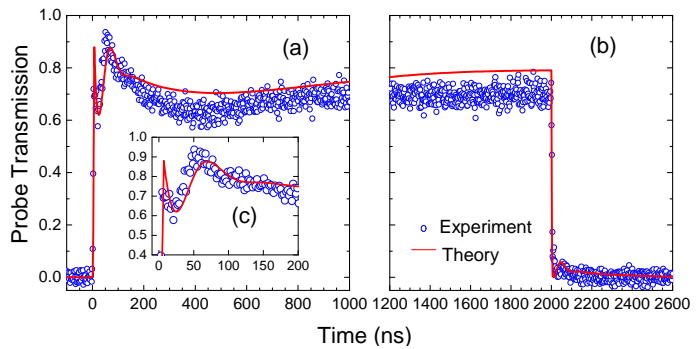

Fig. 3. (color online). Two-photon FIDs with offresonance $(\Delta=2 \pi \times 10 \mathrm{MHz})$ EIT excited by a weak square probe pulse at $\alpha_{0} L=2$ and $\Omega_{c}=1.4 \gamma_{13}$.

tions last for $300 \mathrm{~ns}$ following the rising edge. However, these oscillations are hardly observed in the experimental data after $t>100 \mathrm{~ns}$. For the off-resonance EIT case in Fig. 3, the mismatching also occurs as $t>200$ ns. Besides the detection noise, the degradation of the measured two-photon coherence time may result from the multi-Zeeman-state effect, inhomogeneous broadening caused by the remaining magnetic field, and laser frequency drift during the average time, which can not be easily counted by the simple three-state EIT model.

The work was supported by a grant from the Research Grants Council of the Hong Kong Special Administrative Region, China (Project No. HKUST600809). Some equipment used in the experiment were provided by the Joyce M. Kuok Lasers and Photonics Laboratory and the William Mong Clusters Laboratory.

\section{References}

1. F. Bloch, "Nuclear induction," Phys. Rev. 70, 460 (1946).

2. R. G. Brewer, R. L. Shoemaker, "Photo echo and optical nutation in molecules," Phys. Rev. Lett 27,631 (1971).

3. R. G. Brewer, R. L. Shoemaker, "Optical free induction decay," Phys. Rev. A 6,2001 (1972).

4. D. Grischkowsky, M. M. T. Loy, and P. F. Liao, "Adiabatic following model for two-photon transitions: Nonlinear mixing and pulse propagation," Phys. Rev. A 12, 2514 (1975).

5. M. M. T. Loy, "Observation of two-photon optical nutation and free-induction decay," Phys. Rev. Lett. 36, 1454 (1976).

6. P.F. Liao, J. E. Bjorkholm, and J. P. Gordon, "Observation of two-photon optical free-induction decay in atomic sodium vapor," Phys. Rev. Lett. 39, 15 (1977).

7. D. G. Gold and E. L. Hahn, "Two-photon transient phenomena," Phys. Rev. A 16, 324 (1977).

8. H. W. H. Lee and J. E. Wessel, "Obseravtion of dressedatom effects in three-level free-induction decay," Phys. Rev. Lett. 59, 1416 (1987).

9. K. Toyoda, Y. Takahashi, K. Ishikawa, and T. Yabuzaki, "Optical free-induction decay of laser-cooled ${ }^{85} \mathrm{Rb}$," Phys. Rev. A 56, 1564 (1997).

10. U. Shim, S. Cahn, A. Kumarakrishnan, T. Sleator and J.-T. Kim, "Optical free induction decay in cold ${ }^{85} \mathrm{Rb}$ Atoms," J. Appl. Phys. 41, 3688 (2002). 
11. J. F. Chen, S. Wang, D. Wei, M. M. T. Loy, G. K. L. Wong, and S. Du, "Optical coherent transients in cold atoms: from free-induction decay to optical precursors," Phys. Rev. A (to be published).

12. S. E. Harris, "Electromagnetically induced transparency," Phys. Today 50 (7), 36 (1997).

13. D. Wei, J. F. Chen, M. M. T. Loy, G. K. L. Wong, and S. Du, "Optical precursors with electromagnetically induced transparency in cold atoms," Phys. Rev. Lett. 103, 093602 (2009).

14. M. Fleischhauer, A. Imamoglu, and J. P. Marangos, "Electromagnetically induced transparency: Optics in coherent media," Rev. Mod. Phys, 77, 633 (2005). 